\title{
Association of NAT1 and NAT2 genes with nonsyndromic cleft lip and palate
}

\author{
TAO SONG, DI WU, YONGQIAN WANG, HAIDONG LI, NINGBEI YIN and ZHENMIN ZHAO \\ Center of Cleft Lip and Palate, Plastic Surgery Hospital, Chinese Academy of Medical Sciences \\ and Peking Union Medical College, Beijing 100144, P.R. China
}

Received December 6, 2012; Accepted April 24, 2013

DOI: $10.3892 / \mathrm{mmr} .2013 .1467$

\begin{abstract}
Nonsyndromic cleft lip and palate (NSCLP) is a common congenital deformity, often associated with environmental risk factors, including alcohol, smoking, drugs and radiation exposure. $\mathrm{N}$-acetyltransferase (NAT)1 and NAT2 genes are involved in the detoxification and metabolic activation of numerous drugs and chemicals. The aim of the present study was to investigate whether genetic variations in these two genes and gene-gene interactions are associated with NSCLP. We investigated eight NAT1 tag single nucleotide polymorphisms (SNPs) and five NAT2 tag SNPs, selected from HapMap data. These SNPs were examined for associations with NSCLP in 204 patients and 226 controls. Strong evidence of an association with NSCLP was identified for rs4921580 in the NAT1 gene, and haplotype analysis supported these findings. We also found a significant difference between NSCLP and control groups for rs1041983 in the NAT2 gene. The results of gene-gene interaction analyses also indicated that the combination of rs4921580 $(\mathrm{Cg}+\mathrm{gg}) \times \mathrm{rs} 1041983(\mathrm{Ct}+\mathrm{tt})$ increased the risk of NSCLP. Thus, the present study provides evidence for the role of NAT1 and NAT2 variations in NSCLP, and indicates that interactions between the NAT1 and NAT2 genes may be important in susceptibility to NSCLP.
\end{abstract}

Correspondence to: Dr Ningbei Yin or Dr Zhenmin Zhao, Center of Cleft Lip and Palate, Plastic Surgery Hospital, Chinese Academy of Medical Sciences and Peking Union Medical College, 33 Badachu Road, Shijingshan, Beijing 100144, P.R. China

E-mail: yinningbei@yahoo.com.cn

E-mail: zhaozhenmin0098@vip.sina.com

Abbreviations: NSCLP, nonsyndromic cleft lip with or without cleft palate; NAT, N-acetyltransferase; HWE, Hardy-Weinberg equilibrium; LD, linkage disequilibrium; MAF, minor allele frequency; OR, odds radio; CI, confidence interval

Key words: cleft lip, NAT1, NAT2

\section{Introduction}

Cleft lip with or without palate (CL/P) is one of the most common birth defects, affecting $0.5-1 \%$ of newborns worldwide. Nonsyndromic CL/P (NSCLP) accounts for $70 \%$ of $\mathrm{CL} / \mathrm{P}$ cases, and is genetically distinct from syndromic CL/P. NSCLP is a complex disorder with a multifactorial etiology that involves genetic and environmental factors (1). Alcohol consumption (2), maternal active smoking (3), passive smoking (4), anti-epileptic drugs (5) and radiation exposure (6) are considered to be environmental risk factors for NSCLP; however, the biological mechanisms remain unknown. A number of studies have shown that the risk of NSCLP is associated with variants of genes that regulate detoxification pathways (7).

The human arylamine $\mathrm{N}$-acetyltransferases, NAT1 and NAT2, are important xenobiotic-metabolizing enzymes involved in the detoxification and metabolic activation of numerous drugs and chemicals. NATs have been identified to transfer an acetyl group from acetyl-CoA to the amino group of aryl hydrazines (including isoniazid and hydralazine) or arylamines (including p-aminosalicylate) (8). NAT1 and NAT2 are enzymatically distinct, with certain aromatic amine or hydrazine drugs being preferentially acetylated by NAT1 (e.g., p-aminosalicylic acid and p-aminobenzoic acid) and others by NAT2 (e.g., isoniazid and sulfamethazine). Human NAT1 is ubiquitously expressed at various stages of development (9). NAT1 protein and/or mRNA have been identified in every fetal and adult tissue examined to date, from as early as the blastocyst stage of development (10); NAT2 activity has also been reported in the placenta (11). Folic acid is an essential vitamin in humans and is important in the prevention of neural tube defects. Several studies have shown that NATI is inhibited by folic acid (12). Folic acid is catabolized to $\mathrm{p}$-aminobenzoylglutamate (pABG), which is an endogenous substrate for arylamine NAT (13). Transgenics for human NAT1 and knockouts for NAT2 have been investigated for susceptibility to Dilantin, hydrocortisone and 6-aminonicotinamide-induced orofacial clefting. Erickson et al (14) found that Nat2 significantly influences teratogen-induced orofacial clefting.

The aim of the present study was to characterize genetic variations in NAT1 and NAT2 genes, and to utilize data from case-control studies to investigate the association of single 
nucleotide polymorphisms (SNPs), haplotypes and gene-gene interactions with the risk of NSCLP in the Chinese population.

\section{Materials and methods}

Study population. A total of 204 NSCLP patients and 226 normal controls were included in this case-control study. Subjects in the control group had no congenital malformations of the body and no family history of genetic disease, were born in the same region as the NSCLP patients and had a male:female ratio that was as similar to that of the NSCLP group as possible. All the study participants were recruited between January 2010 and January 2012 from the Cleft Surgery Department of the Plastic Surgery Hospital, Chinese Academy of Medical Sciences (Beijing, China). Informed consent was obtained from each participant prior to enrollment in the study, and the study was approved by the ethics committee of Plastic Surgery Hospital, Chinese Academy of Medical Sciences, Beijing, China. General characteristics including age, gender, ethnicity, health status and birth defects were documented.

SNP identification and selection. Using the HapMap genome browser (http://www.hapmap.org/cgi-perl/ gbrowse/hapmap3r2_B36), based on the Han Chinese population in Beijing (CHB), eight tag SNPs ( $\mathrm{r}^{2}$ coefficient cut-off, 0.80 ; with a minor allele frequency of 0.05 ) were selected to capture the NAT1 region of chromosome 8. Five tag SNPs were selected for the NAT2 region of chromosome 8 .

Genotyping. Peripheral blood samples $(2 \mathrm{ml})$ were collected from each participant and frozen. Genomic DNA was extracted from a 200- $\mu$ l aliquot of each sample using a Tiangen ${ }^{\text {TM }}$ Genomic DNA kit (Tiangen, Beijing, China) according to the manufacturer's instructions, and was stored at $-70^{\circ} \mathrm{C}$. All the SNPs were genotyped using the Sequenom MassARRAY matrix-assisted laser desorption/ionization time-of-flight mass spectrometry platform (Sequenom, Inc., San Diego, CA, USA). Primers were designed using a semi-automated method (Assay Design 3.1; Sequenom, Inc.); primer sequences are available on request. The call rate for each assay was set at $>90 \%$.

Statistical analysis. Data from the control and NSCLP groups were compared and analyzed using SPSS 17.0 software (SPSS Inc., Chicago, IL, USA), and HWE software (http://www. broad.mit.edu/mpg/haploview/) was used for assessment of the Hardy-Weinberg equilibrium. Based on the multivariable logistic regression method, the case-control association of genotypes in three inheritance models (dominant, recessive and additive) was examined, and these models were coded as follows for genotypes $\mathrm{AA}, \mathrm{AB}$ and $\mathrm{BB}$, respectively (assuming $B$ is the minor allele): dominant 011 ( $A A$ vs. $A B+B B$ ), recessive 001 (AA + AB vs. $\mathrm{BB}$ ) and additive 012 (trend test on $\mathrm{B}$ allele count). The odds ratios (ORs) and $95 \%$ confidence intervals (CIs) were provided. The association between each SNP and haplotype with NSCLP was estimated using Haploview software (http://www.broad.mit.edu/mpg/haploview/). For haplotype construction, genotype data from the case and control groups were used to estimate intermarker linkage disequilibrium (LD) by measuring pairwise $\mathrm{D}^{\prime}$ and $\mathrm{r}^{2}$, and defining LD blocks. We used the CI method in the Haploview software to define an LD block with an extended spine when $\mathrm{r}^{2}$ was 0.8 . P-values were corrected for multiple tests with 10,000 permutations. To identify higher order gene-gene interactions in our samples, we used logistic regression analysis to calculate ORs and 95\% CIs to search for gene-gene interactions in the case and control groups. The interactions between SNPs were classified in four groups using a dominant model. In each case, a wild-type genotype was evaluated as a reference. Since the research is exploratory and Bonferroni corrections are too conservative, Bonferroni corrections were not used.

\section{Results}

Study subject characteristics. A total of 13 SNPs were genotyped from NSCLP patients and normal controls of a Han Chinese origin. The mean age was $3.23 \pm 0.91$ years old and the male:female gender ratio was 1:0.76 in the NSCLP group, while the mean age was $3.96 \pm 1.03$ years old and the male:female gender ratio was 1:0.70 in the control group. The genomic position, nucleic acid composition and minor allele frequencies of the SNPs are shown in Table I. The HWE was calculated for all the SNPs; none of the SNPs among these groups deviated from the HWE.

Single SNP analysis. To evaluate the association between genetic variants and the risk of NSCLP, we compared NAT1 and NAT2 genotype frequency distributions in the NSCLP and control groups (Table II). For NAT1, there was a significant difference in the allele frequency of rs 4921580 between the NSCLP and control groups $(\mathrm{P}=0.0128)$; however, no significant difference was identified following a correction with 10,000 permutations ( $\mathrm{P}=0.0872)$. The dominant model and Cochran-Armitage trend tests of rs4921580 demonstrated a significant difference ( $\mathrm{P}=0.010$ and 0.034 , respectively). For NAT2, a Cochran-Armitage trend test of rs1041983 remained significant $(\mathrm{P}=0.040)$ between the NSCLP and control groups.

Haplotype analysis. For NAT1, the Haploview program revealed that rs2410545, rs4921580 and rs17693097 were in the same LD block (Fig. 1A). Haplotype analysis of polymorphisms in the NAT1 gene showed that SNP combinations were associated with the risk of NSCLP (Table III). The GGG haplotypes were shown to have an increased risk of NSCLP (OR, 2.832; 95\% CI, 1.437-5.584; $\mathrm{P}=0.0093$ ), which remained significant after correction with 10,000 permutations $(\mathrm{P}=0.0191)$. For NAT2, rs11996129 and rs1961456 were in the same LD block (block 1), while rs1041983 and rs1799931 were also in the same LD block (block 2; Fig. 1B; Table III). There was no difference at block 1 or block 2 between the NSCLP and controls groups.

Gene-gene interaction analysis. A logistic regression model was built to analyze rs4921580 in NAT1 and rs1041983 in NAT2. The combinations of rs4921580 (Cg+gg) x rs1041983 $(\mathrm{Ct}+\mathrm{tt})$ were significantly associated with NSCLP (OR, 1.983; 95\% CI, 1.150-3.418; P=0.014; Table IV).

\section{Discussion}

The results of the present study revealed a significant association of NSCLP with rs4921580 in the NAT1 gene and 
Table I. Characteristics of NAT1 and NAT2 gene polymorphisms.

\begin{tabular}{|c|c|c|c|c|c|c|}
\hline Gene symbol & rs number & SNP function & Location & Alleles $^{\mathrm{a}}$ & MAF & P-HWE \\
\hline \multirow[t]{8}{*}{ NAT1 } & rs2410545 & UTR-5 & Chr8 18068835 & $\mathrm{a} / \mathrm{G}$ & 0.412 & 0.1809 \\
\hline & rs4921580 & Intron & Chr8 18071000 & $\mathrm{C} / \mathrm{g}$ & 0.174 & 0.8829 \\
\hline & rs 17693097 & Intron & Chr8 18072809 & $\mathrm{c} / \mathrm{G}$ & 0.113 & 0.9299 \\
\hline & rs 13278990 & Intron & Chr8 18073354 & $\mathrm{a} / \mathrm{T}$ & 0.328 & 0.4586 \\
\hline & rs6586714 & Intron & Chr8 18073942 & $\mathrm{a} / \mathrm{G}$ & 0.087 & 1 \\
\hline & rs 17126350 & Intron & Chr8 18074322 & $\mathrm{~A} / \mathrm{g}$ & 0.047 & 0.7727 \\
\hline & rs4921880 & Intron & Chr8 18075906 & $\mathrm{a} / \mathrm{T}$ & 0.45 & 0.0651 \\
\hline & rs8190845 & Intron & Chr8 18078628 & $\mathrm{a} / \mathrm{G}$ & 0.037 & 0.218 \\
\hline \multirow[t]{5}{*}{ NAT2 } & rs11780272 & Intron & Chr8 18249652 & $\mathrm{c} / \mathrm{T}$ & 0.033 & 1 \\
\hline & rs11996129 & Intron & Chr8 18254575 & $\mathrm{c} / \mathrm{T}$ & 0.224 & 0.4344 \\
\hline & rs1961456 & Intron & Chr8 18255709 & $\mathrm{a} / \mathrm{G}$ & 0.297 & 0.8645 \\
\hline & rs 1041983 & cds-synon & Chr8 18257795 & $\mathrm{C} / \mathrm{t}$ & 0.385 & 0.6728 \\
\hline & rs1799931 & Missense & Chr8 18258370 & $\mathrm{a} / \mathrm{G}$ & 0.159 & 1 \\
\hline
\end{tabular}

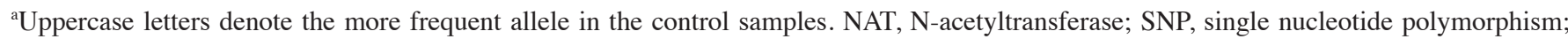
MAF, minor allele frequency; HWE, Hardy-Weinberg equilibrium.

A

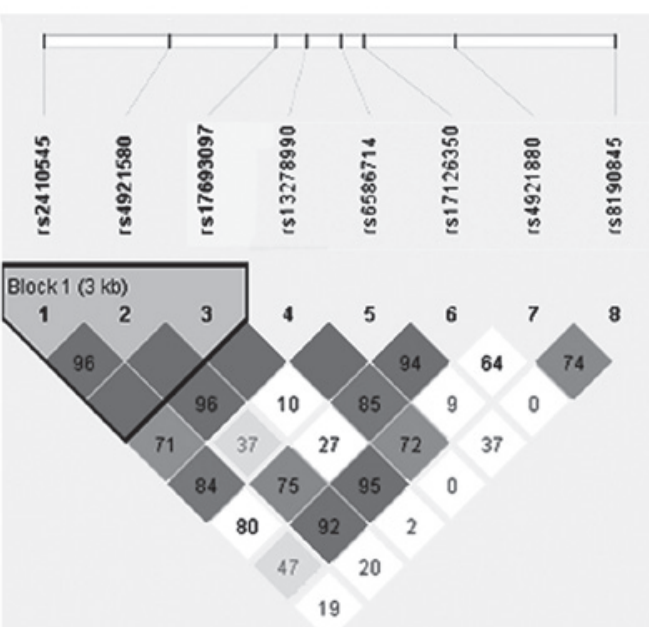

B
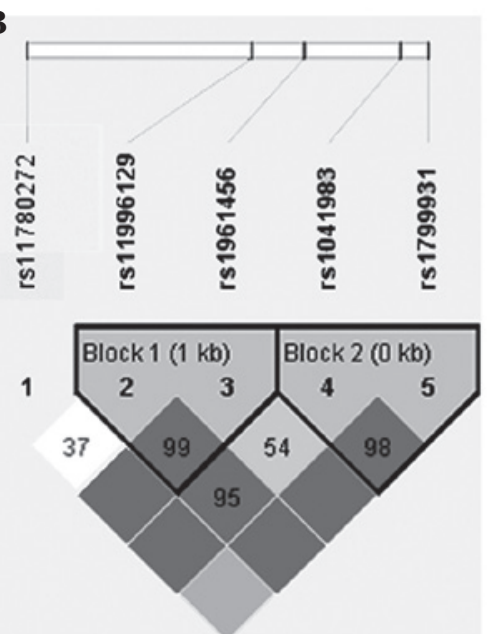

Figure 1. Linkage disequilibrium (LD) pattern for NAT1 and NAT2 genes. Haplotype block structure is shown, as depicted by Haploview. The value within each diamond represents the pairwise correlation between the single nucleotide polymorphisms (SNPs; measured as D'). Diamonds without a number correspond to $\mathrm{D}^{\prime}=1$. Shading represents the magnitude and significance of pairwise LD, with a black-to-white gradient reflecting higher to lower $\mathrm{D}^{\prime}$ values. (A) NAT1; (B) NAT2.

rs1041983 in the NAT2 gene. For the NAT1 gene, the GGG haplotype, including rs2410545, rs4921580 and rs17693097, was significantly associated with NSCLP. Analysis using a logistic regression model also showed that gene-gene interactions between NAT1 and NAT2 increased the risk of NSCLP.

Previous studies on NAT1 mainly focused on rs1057126 (T1088A) and rs15561 (C1095A). In a Californian population-based case-control study, the OR for isolated CL/P was increased ( $\mathrm{OR}=1.7 ; 95 \% \mathrm{CI}=0.97-2.9)$ among infants homozygous for 1095A compared with genotype 1095CC, while not for heterozygotes. There was an $\sim 4$-fold increased risk for isolated CL/P for the 1088AA genotype in the presence of maternal smoking. Similar to the 1088 polymorphism analysis, the AA genotype of the NAT1 1095 polymorphism modified risks for isolated $\mathrm{CL} / \mathrm{P}$ and was associated with smoking with a 4-fold increased risk. The risk with the NAT1 genotype 1088AA + 1095AA in the presence of maternal smoking was higher compared with 1088TT + 1095CC (15). A study on NAT1 1095 genotypes by Lammer et al (16) found a 2-fold higher risk for isolated CL/P among infants who were homozygous for the variant allele and whose mothers did not take multivitamins during early pregnancy. However, there were not any differential risks for clefts associated with maternal multivitamin consumption for the NAT1 1088 genotypes. Lie et al (17) did not identify an association between NAT1 and CL.

For NAT2, children heterozygous for the rs1799930 (G590A) variant were shown to have a 2-fold risk of NSCLP. Furthermore, the C-A-G haplotype of NAT2 (referring to the variants of the SNPs rs1799929/C481T, rs1799930 and rs1799931/G857A, respectively) has been associated with 


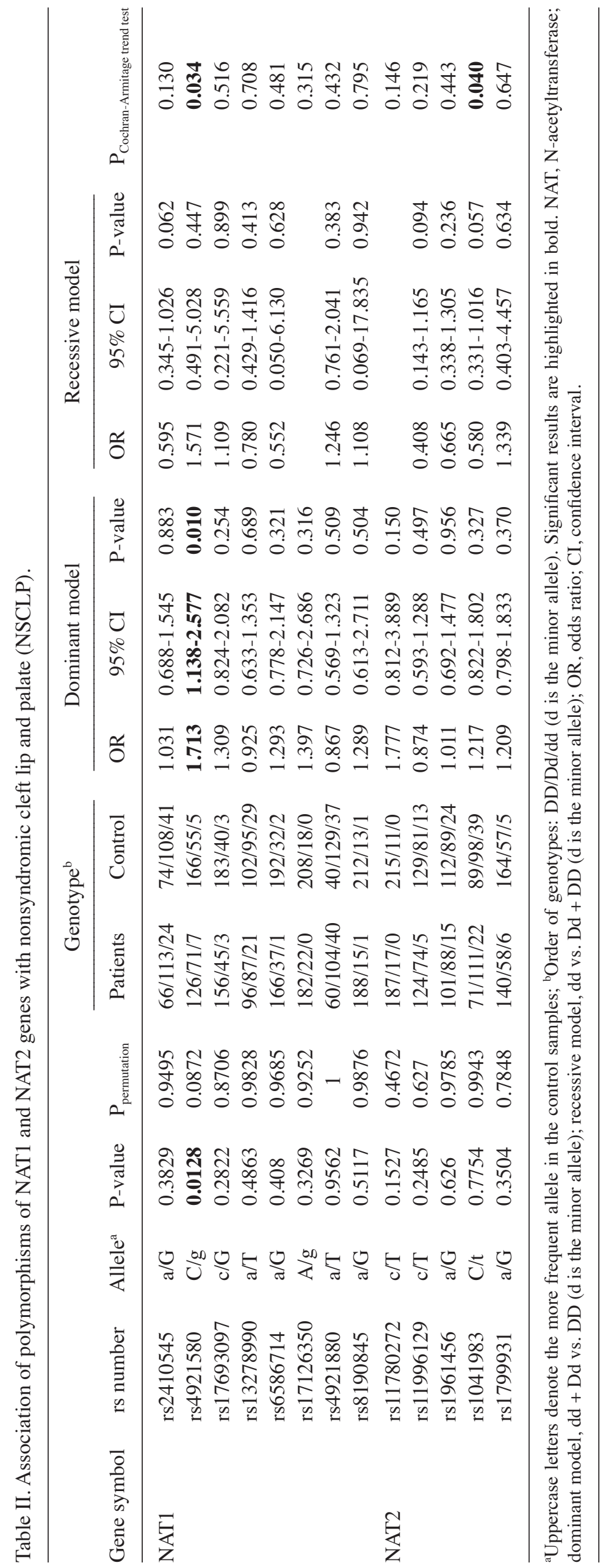


Table III. Haplotype analysis of NAT1 and NAT2 gene polymorphisms.

\begin{tabular}{|c|c|c|c|c|c|c|c|c|}
\hline \multirow[b]{2}{*}{ Gene symbol } & \multirow[b]{2}{*}{ Haplotype } & \multicolumn{2}{|c|}{ Frequency } & \multirow[b]{2}{*}{ OR } & \multicolumn{2}{|c|}{$95 \% \mathrm{CI}$} & \multirow[b]{2}{*}{ Nominal P-value } & \multirow[b]{2}{*}{ Permuted P-value } \\
\hline & & Case & Control & & $95 \%$ Low & 95\% High & & \\
\hline \multirow[t]{4}{*}{ NAT1 } & GCG & 0.399 & 0.436 & 1 & Ref & Ref & 0.2735 & 0.5209 \\
\hline & $\mathrm{ACG}$ & 0.393 & 0.421 & 1.041 & 0.7731 & 1.401 & 0.41 & 0.7265 \\
\hline & GGC & 0.125 & 0.102 & 1.349 & 0.8599 & 2.115 & 0.2822 & 0.53 \\
\hline & GGG & 0.080 & 0.039 & 2.832 & 1.437 & 5.584 & 0.0093 & 0.0191 \\
\hline \multicolumn{9}{|l|}{ NAT2 } \\
\hline \multirow[t]{3}{*}{ Block 1} & $\mathrm{TG}$ & 0.708 & 0.694 & 1 & Ref & Ref & 0.6573 & 1.0000 \\
\hline & $\mathrm{CA}$ & 0.205 & 0.24 & 0.8394 & 0.6039 & 1.167 & 0.2217 & 0.5009 \\
\hline & TA & 0.084 & 0.066 & 1.262 & 0.7496 & 2.125 & 0.3026 & 0.6667 \\
\hline \multirow[t]{3}{*}{ Block 2} & $\mathrm{CG}$ & 0.620 & 0.608 & 1 & Ref & Ref & 0.7276 & 1.0000 \\
\hline & TG & 0.209 & 0.244 & 0.8367 & 0.6011 & 1.165 & 0.2223 & 0.5046 \\
\hline & $\mathrm{TA}$ & 0.171 & 0.146 & 1.157 & 0.7929 & 1.688 & 0.3068 & 0.6806 \\
\hline
\end{tabular}

Significant results are highlighted in bold. NAT, N-acetyltransferase; OR, odds ratio; CI, confidence interval; Ref, reference. The highestfrequency haplotype in the control group is used as reference.

Table IV. Results of gene-gene interactions using a logistic regression method.

\begin{tabular}{llllr}
\hline & \multicolumn{2}{c}{ Frequency } & & \\
\cline { 2 - 3 } SNP in gene (NAT1 x NAT2) & Cases & Controls & OR & 95\% CI \\
\hline rs4921580 x rs1041983 & & & & Reference \\
CC x CC & 0.25 & 0.300885 & 1 & $0.623-2.588$ \\
Cg+gg x CC & 0.098039 & 0.09292 & 1.270 & $0.637-1.635$ \\
CC x Ct+tt & 0.367647 & 0.433628 & 1.020 & 0.933 \\
Cg+gg x Ct+tt & 0.284314 & 0.172566 & 1.983 & $1.150-3.418$ \\
\hline
\end{tabular}

Significant results are highlighted in bold. NAT, N-acetyltransferase; OR, odds ratio; CI, confidence interval. A wild type genotype is used as a reference.

isolated CL in a Norwegian population. However, there was no indication of interaction between the NAT2 C-A-G haplotype in children and maternal smoking (17). In case-parent triads, the overtransmission of NAT2 G590A was observed in an oral cleft group, while not in the control group (18). A number of additional studies did not find an association between NAT2 and NSCLP $(15,19)$

To the best of our knowledge, this is the first study on the association of NAT1 and NAT2 genes with NSCLP using tagging SNP methods, and also the first study on a Chinese population. For NAT1, the results of the present study revealed that rs4921580 was associated with NSCLP, which has not been previously reported. Additionally, the rs2410545-rs4921580-rs17693097 haplotype (GGG) was found to have a 2.8-fold risk for NSCLP. According to the HapMap CHB database, rs15561 is located in the same LD block as rs4921880. However, an association between rs4921880 and NSCLP was not identified. This may be due to the use of different geographical populations, sample sizes or study methods. For NAT2, rs1041983 was found to be associ- ated with NSCLP, which has not been previously reported. rs1041983 function is synonymous (cds-synon); no change occurs in the amino acid sequence of the protein. DNA mutations do not result in a change in the amino acid sequence of a protein; however, a recent study showed that the 'silent' polymorphism may lead to the synthesis of a protein product with the same amino acid sequence but with different structural and functional properties (20). Therefore, rs1041983 should not be neglected and further studies are required. rs1799930 is located in the same LD block as rs11996129 in the HapMap CHB database. However, an association between rs11996129 and NSCLP was not identified. rs1799931 was not shown to be associated with NSCLP, which was consistent with the results of Lie et al (17).

NAT1 and NAT2 are enzymatically distinct, with certain aromatic amines being preferentially acetylated by NAT1 (e.g., p-aminosalicylic acid and p-aminobenzoic acid) and others by NAT2 (e.g., isoniazid and sulfamethazine). Cornish et al (21) confirmed that NAT activity levels are strictly gene-dose-dependent and that NAT deficiency is not 
compensated for by other enzymes of xenobiotic metabolism. The deficiency of both NAT1 and NAT2 may impair the detoxification and metabolic activation of drugs (22). In the present study, the combination of rs4921580 (Cg+gg) x rs1041983 $(\mathrm{C} t+\mathrm{tt})$ was shown to increase the risk of NSCLP, which has not been previously reported. A possible explanation for this result may be that the mutation of the two genes results in metabolic abnormalities that are associated with NSCLP. Erickson et al (14) found that Nat2 significantly influenced teratogen-induced orofacial clefting using transgenic and knockout animals. However, Sugamori et al (22) showed that Nat1/Nat2 double-knockout mice develop normally and do not suffer from any obvious phenotypic defects. This indicates that the mechanism of NAT1 and NAT2 requires further investigation.

The present study had several limitations. Firstly, the sample size used was relatively small and other ethnic populations were not included. Secondly, NAT1 and NAT2 are associated with the detoxification and metabolic activation of numerous chemicals. In the present study, environmental factors during pregnancy, including alcohol consumption, maternal active smoking, passive smoking, anti-epileptic drugs and radiation exposure, were not investigated. Therefore, further studies are required.

In conclusion, despite limitations caused by the use of a small sample size, evidence in support of the association of rs4921580 in the NAT1 gene and rs1041983 in the NAT2 gene with NSCLP in a Chinese population was identified; haplotype analysis of the gene supported these findings, and gene-gene interactions between NAT1 and NAT2 were also demonstrated to play a role in the susceptibility to NSCLP. Further studies are required to investigate the association between NAT1 and NAT2, and NSCLP.

\section{Acknowledgements}

The authors thank all the participants who donated samples for this study. This study was supported by the National Natural Science Foundation of China (30901569).

\section{References}

1. Stuppia L, Capogreco M, Marzo G, et al: Genetics of syndromic and nonsyndromic cleft lip and palate. J Craniofac Surg 22: 1722-1726, 2011.

2. Munger RG, Romitti PA, Daack-Hirsch S, Burns TL, Murray JC and Hanson J: Maternal alcohol use and risk of orofacial cleft birth defects. Teratology 54: 27-33, 1996.

3. Little J, Cardy A, Arslan MT, Gilmour M and Mossey PA: Smoking and orofacial clefts: a United Kingdom-based case-control study. Cleft Palate Craniofac J 41: 381-386, 2004.

4. Jia ZL, Shi B, Chen CH, Shi JY, Wu J and Xu X: Maternal malnutrition, environmental exposure during pregnancy and the risk of non-syndromic orofacial clefts. Oral Dis 17: 584-589, 2011.
5. Hernández-Diaz S, Smith CR, Shen A, et al: Comparative safety of antiepileptic drugs during pregnancy. Neurology 78: 1692-1699, 2012.

6. Cech I, Burau KD and Walston J: Spatial distribution of orofacial cleft defect births in Harris County, Texas, 1990 to 1994, and historical evidence for the presence of low-level radioactivity in tap water. South Med J 100: 560-569, 2007.

7. Ramirez D, Lammer EJ, Iovannisci DM, Laurent C, Finnell RH and Shaw GM: Maternal smoking during early pregnancy, GSTP1 and EPHX1 variants, and risk of isolated orofacial clefts. Cleft Palate Craniofac J 44: 366-373, 2007.

8. Sim E, Fakis G, Laurieri N and Boukouvala S: Arylamine $\mathrm{N}$-acetyltransferases - from drug metabolism and pharmacogenetics to identification of novel targets for pharmacological intervention. Adv Pharmacol 63: 169-205, 2012.

9. Mortensen HM, Froment A, Lema G, et al: Characterization of genetic variation and natural selection at the arylamine $\mathrm{N}$-acetyltransferase genes in global human populations. Pharmacogenomics 12: 1545-1558, 2011.

10. Boukouvala S and Fakis G: Arylamine N-acetyltransferases: what we learn from genes and genomes. Drug Metab Rev 37: 511-564, 2005.

11. Smelt VA, Mardon HJ and Sim E: Placental expression of arylamine $\mathrm{N}$-acetyltransferases: evidence for linkage disequilibrium between NAT $1 * 10$ and NAT2 $* 4$ alleles of the two human arylamine N-acetyltransferase loci NAT1 and NAT2. Pharmacol Toxicol 83: 149-157, 1998.

12. Ward A, Hickman D, Gordon JW and Sim E: Arylamine $\mathrm{N}$-acetyltransferase in human red blood cells. Biochem Pharmacol 44: 1099-1104, 1992.

13. Minchin RF: Acetylation of p-aminobenzoylglutamate, a folic acid catabolite, by recombinant human arylamine $\mathrm{N}$-acetyltransferase and U937 cells. Biochem J 307 (Pt 1): 1-3, 1995.

14. Erickson RP, Cao W, Acuña DK, et al: Confirmation of the role of $\mathrm{N}$-acetyltransferase 2 in teratogen-induced cleft palate using transgenics and knockouts. Mol Reprod Dev 75: 1071-1076, 2008.

15. Lammer EJ, Shaw GM, Iovannisci DM, Van Waes J and Finnell RH: Maternal smoking and the risk of orofacial clefts: susceptibility with NAT1 and NAT2 polymorphisms. Epidemiology 15: 150-156, 2004.

16. Lammer EJ, Shaw GM, Iovannisci DM and Finnell RH: Periconceptional multivitamin intake during early pregnancy, genetic variation of acetyl-N-transferase 1 (NAT1), and risk for orofacial clefts. Birth Defects Res A Clin Mol Teratol 70: 846-852, 2004.

17. Lie RT, Wilcox AJ, Taylor J, et al: Maternal smoking and oral clefts: the role of detoxification pathway genes. Epidemiology 19: 606-615, 2008

18. Shi M, Christensen K, Weinberg CR, et al: Orofacial cleft risk is increased with maternal smoking and specific detoxification-gene variants. Am J Hum Genet 80: 76-90, 2007.

19. van Rooij IA, Groenen PM, van Drongelen M, Te Morsche RH, Peters WH and Steegers-Theunissen RP: Orofacial clefts and spina bifida: $\mathrm{N}$-acetyltransferase phenotype, maternal smoking, and medication use. Teratology 66: 260-266, 2002.

20. Komar AA: Silent SNPs: impact on gene function and phenotype. Pharmacogenomics 8: 1075-1080, 2007.

21. Cornish VA, Pinter K, Boukouvala S, et al: Generation and analysis of mice with a targeted disruption of the arylamine $\mathrm{N}$-acetyltransferase type 2 gene. Pharmacogenomics J 3: 169-177, 2003.

22. Sugamori KS, Wong S, Gaedigk A, et al: Generation and functional characterization of arylamine $\mathrm{N}$-acetyltransferase Nat1/Nat2 double-knockout mice. Mol Pharmacol 64: 170-179, 2003. 